\title{
Real-time Java simulations of multiple interference dielectric filters ${ }^{\text {th }}$
}

\author{
Alexandre N. Kireev*, Olivier J.F. Martin \\ Nanophotonics and Metrology Laboratory, Swiss Federal Institute of Technology Lausanne (EPFL), Switzerland
}

\section{A R T I C L E I N}

\section{Article history:}

Received 23 January 2008

Received in revised form 20 June 2008

Accepted 20 July 2008

Available online 20 August 2008

\section{PACS:}

42.79.Ci

42.82.-m

77.55. $+\mathrm{f}$

Keywords:

Thin films

Optical properties

Filters

Interference

\begin{abstract}
A B S T R A C T
An interactive Java applet for real-time simulation and visualization of the transmittance properties of multiple interference dielectric filters is presented. The most commonly used interference filters as well as the state-of-the-art ones are embedded in this platform-independent applet which can serve research and education purposes. The Transmittance applet can be freely downloaded from the site http://cpc.cs.qub.ac.uk.
\end{abstract}

\section{Program summary}

Program title: Transmittance

Catalogue identifier: AEBQ_v1_0

Program summary URL: http://cpc.cs.qub.ac.uk/summaries/AEBQ_v1_0.html

Program obtainable from: CPC Program Library, Queen's University, Belfast, N. Ireland

Licensing provisions: Standard CPC licence, http://cpc.cs.qub.ac.uk/licence/licence.html

No. of lines in distributed program, including test data, etc.: 5778

No. of bytes in distributed program, including test data, etc.: 90474

Distribution format: tar.gz

Programming language: Java

Computer: Developed on PC-Pentium platform

Operating system: Any Java-enabled OS. Applet was tested on Windows ME, XP, Sun Solaris, Mac OS

RAM: Variable

Classification: 18

Nature of problem: Sophisticated wavelength selective multiple interference filters can include some tens or even hundreds of dielectric layers. The spectral response of such a stack is not obvious. On the other hand, there is a strong demand from application designers and students to get a quick insight into the properties of a given filter.

Solution method: A Java applet was developed for the computation and the visualization of the transmittance of multilayer interference filters. It is simple to use and the embedded filter library can serve educational purposes. Also, its ability to handle complex structures will be appreciated as a useful research and development tool.

Running time: Real-time simulations

(c) 2008 Elsevier B.V. All rights reserved.

\section{Introduction}

Wavelength selective multiple interference filters are widespread in science and technology [1,2]. Their operation principles are well understood and implemented in commercial software. So-

\footnotetext{
This paper and its associated computer program are available via the Computer Physics Communications homepage on ScienceDirect (http://www. sciencedirect.com/science/journal/00104655).

* Corresponding author.

E-mail addresses: alexandre.kireev@epfl.ch (A.N. Kireev), olivier.martin@epfl.ch (O.J.F. Martin).

URL: http://nam.epfl.ch/ (O.J.F. Martin).
}

phisticated filters can include some tens or even hundreds of dielectric layers. Generally, before optimization, the spectral response of such a stack is not obvious. Furthermore, there are no common recipes for a filter design with specific properties. Therefore the design of interference filters remains a vivid research topic [3-5]. On the other hand, there is also a strong demand from application designers and students to get a quick insight into the properties of a given multilayer interference filter.

In this paper, we describe a Java applet for the computation and the visualization of the transmittance of multilayer interference filters. The applet was developed with two objectives in mind: on one side, its interface is easy to use and the embedded filter library can serve educational purposes. On the other side, its efficiency 
and ability to handle complex multilayer structures make it a useful research and development tool. The Transmittance applet has been tested on major operating systems.

Java applets provide a versatile framework for interactive visualization, networking, database connectivity and on-line numerical simulations [6,7]. Their platform independence, extended graphical capabilities, and seamless integration of graphical user interface (GUI) components represent very attractive features for the enduser. Developers appreciate the object-oriented structure of Java and the absence of pointers facilitates the creation of large applications and their debugging. Finally the Java applets improve considerably the appearance of Web pages and increase their functionality $[8,9]$. Recent progress in just-in-time (JIT) technologies and dramatic increase of the raw CPU power have made Java applets suitable for complicated numerical modeling and extended run-time simulations [10].

The paper is organized as follows: Section 2 introduces the Transmittance applet and describes the mathematical formalism based on the use of transfer matrix. In Section 3 we give examples of canonical interference filters available for interactive visualization and experimentation.

\section{Transmittance applet}

Typical interference filters are composed of several spacer regions surrounded by multilayer reflectors [1]. The reflector regions consist of alternating layers $H$ and $L$ of dielectrics with high, respectively low refractive indices. The optical thickness of each layer $j$ is one quarter of the central wavelength $\lambda_{j}=\lambda_{0} / n_{j}$ inside this layer, so that light interfere destructively. The central wavelength in vacuum is $\lambda_{0}$ and $n_{j}$ the refractive index of layer $j$. The optical thickness of the spacers, also known as cavities, is half the central wavelength. The combined action of these basic components produces the filter's band-pass spectrum. Interference filters can include several hundreds of layers or can contain layers with gradual variations of optical index.

The transfer matrix formalism is the most suitable method for modeling the optical properties of dielectric stacks. It describes propagation of electromagnetic fields in a stratified medium, composed of $N$ dielectric layers with material parameters $\left(\varepsilon_{j}, \mu_{j}\right)$, as depicted on Fig. 1. A detailed description can be found in Refs. $[1,2]$ and we shall only outline its main features here.

Taking into account the translational invariance along the $x$-axis and superposition of forward and backward propagating waves with amplitudes $a_{j}, b_{j}$ along the $z$-axis, the electromagnetic field is factorized inside each layer $j$. It suffices to consider only two fundamental polarizations of light, transversal electric (TE) and transversal magnetic (TM) waves. The applet considers only normal incidence, where both polarizations are degenerate. If $k_{0}=2 \pi / \lambda_{0}$ and $n_{j}^{2}=\varepsilon_{j} \mu_{j}$ then the full transfer matrix $\widehat{S}$ relates the input and output field amplitudes of the complete stack. The stack reflectance is found as $R=\left|b_{\text {in }} / a_{\text {in }}\right|^{2}$ and the stack transmittance as $T=\left(n_{\text {out }} / n_{\text {in }}\right)\left|a_{\text {out }} / a_{\text {in }}\right|^{2}$.

The development of Web-based applications requires both a precise mathematical modeling and a transparent interface. The underlying physical phenomenon must be important for applications and difficult to understand without numerical simulations. Java applets or Web-based applications should allow an efficient control of the mathematical kernel with the help of a limited number of parameters and provide easy access to the simulation results and their visualization.

Our Transmittance applet has been written with the purpose of fulfilling all these requirements. It comprises a mathematical kernel based on the transfer matrix formalism and a graphical interface that inputs and outputs simulation data. Special care has been taken to cover a wide range of realistic models and geometries, to

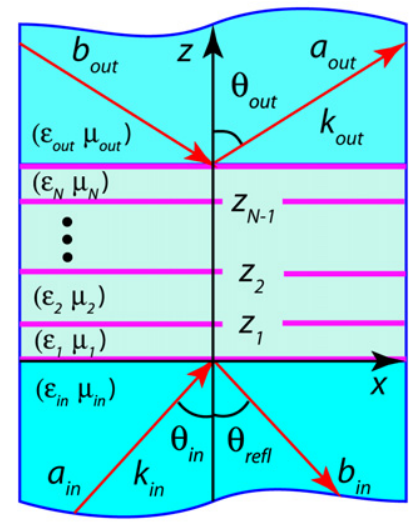

Fig. 1. Stratified medium forming a typical interference filter. The modal structure of electric field is specified by the material parameters $\left(\varepsilon_{j}, \mu_{j}\right)$, while $\theta_{\text {in }}, \theta_{\text {refl }}$ and $\theta_{\text {out }}$ are the angles of incoming, reflected and transmitted light.

facilitate their modification with the help of a limited number of GUI controls and, most of all, to provide an easily understandable graphical presentation of the simulation results.

Visually, the Transmittance applet is composed of three graphical windows, a toolbar with sliders and an Action box (Fig. 2). The left-hand side window shows the spectrum of incident visible light with wavelengths $\lambda_{0}$ between 400 and $750 \mathrm{~nm}$. The modeled stratified optical medium, composed of thin dielectric layers with thicknesses $d_{j}$ and refractive indices $n_{j}$, is shown in the central graphical window. Its spectral response is calculated in real time and appears in the right-hand side graphical window.

Only the most important parameters can be changed with the help of sliders and the selection box. The menu panel gives the possibility to explore several interference filters found in practice, as described in Section 3. Refractive indices can be changed with the mouse in the central window. Any selected filter supposes its own design principle. Hence, the number of layers can only be adjusted in a way compatible with this principle. The central wavelength of the filter can be set in the left graphical window, while the Reset button reverts to the basic configuration of the chosen filter.

Two last options of the Action box give more freedom to experiment with less restrictive multilayer stacks. For periodic stacks, the width of odd and even layers, in addition to their refractive indices, can be adjusted independently using the mouse in the central window. The last option, Design stack, allows the individual adjustment of each layer thickness and refractive index. In that case, the Reset command cycles the filter design through several state-of-the-art filters, description of which can be found in current literature. The grating structure can be inspected interactively, since the Applet shows the $n_{j}$ and $d_{j}$ parameters when hovering the mouse over the layer. The layer thickness is also displayed in units of quarterwavelengths, stressing the importance of this parameter for a filter design.

\section{Interference filters}

The Applet provides several examples of the most important interference filters, like transmittance and reflectance filters, singlecavity Fabry-Perot etalons, dual-cavity filters and some more elaborated filters.

As soon as a filter central wavelength $\lambda_{0}$ (designated as $L_{0}$ in the Applet and shown in the left window) is set, the mathematical kernel reads the filter parameters, scans the visible spectrum in real time and displays the spectrum of transmitted light in the right-hand side window. At start-up the Applet is showing the 


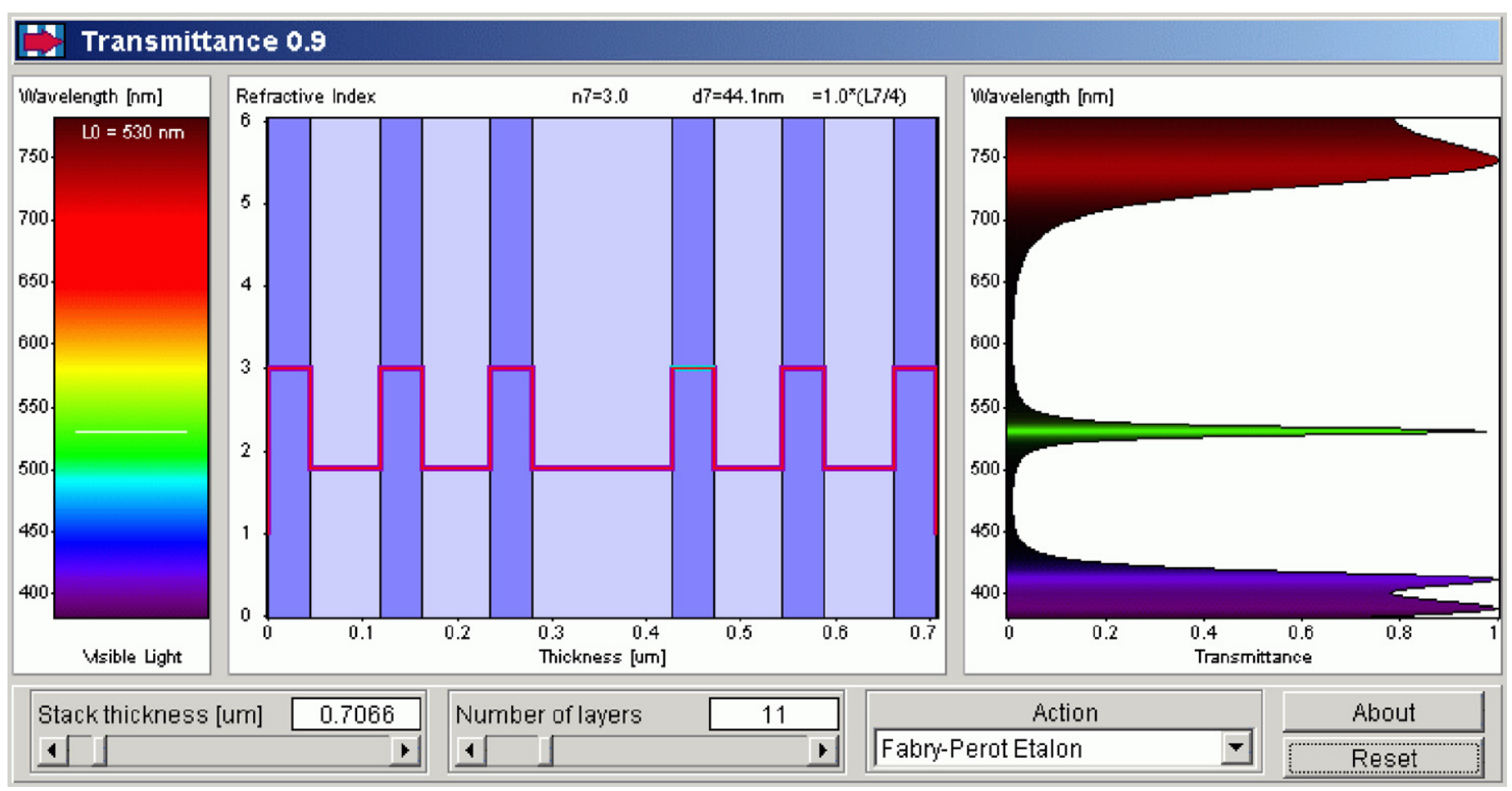

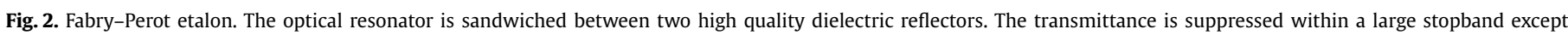
for an extremely narrow interval around $\lambda_{0}$.

Transmittance filter. This configuration can always be restored with the Reset button. Each filter has its own default configuration.

The following gives a short description of the available filter options and the possibilities to modify them. The Action box provides access to one of the six most common filters, two Fabry-Perot etalons, and two less restrictive Periodic and Design stacks.

The following notation is used in the Applet interface:

\begin{tabular}{ll}
\hline$M$ & quarter-wavelength layer with intermediate value of refractive index \\
$A$ & air interface \\
$G$ & glass interface \\
$n_{L}$ & refractive index outside the left interface \\
$L_{j}$ & radiation wavelength inside the layer $j$ \\
\hline
\end{tabular}

\subsection{Transmittance filter}

The Transmittance filter consists of alternating half-wavelength cavities $\mathrm{HH}$ of high index material and quarter-wavelength layers $L$ of low index material. It is described by a sequence $A(H H L)^{k}$ HH $A$, with $k=0,1,2, \ldots$. This filter suppresses light with wavelengths outside of its pass-band. It is resetted to a band-pass filter with $k=3$.

\subsection{Inverted Transmittance filter}

The filter is composed of alternating layers $H$ and half-wavelength cavities $L L$ of low index material. It is described by a sequence $A(H L L)^{k} H A$. The Reset button reconstructs the bandpass configuration with $k=4$.

\subsection{Reflectance filter}

The Reflectance filter consists of alternating dielectric layers $H$ and $L$ with the optical thickness of each layer equal to $\lambda_{j} / 4$. It has a structure $A(H L)^{k} H A$ and suppresses all radiation within its stop-band. The default configuration is A HLHLHLH A.

\subsection{Inverted Reflectance filter}

It is the same as the reflectance filter but with inverted sequence of the layers $H$ and $L$. It is resetted to a beam splitter A LHLHLHLHL A.

\subsection{Fabry-Perot etalon}

The Fabry-Perot etalon consists of two highly-efficient reflectors separated by a dielectric resonator with optical thickness $L L$. The reflectors themselves are the typical dielectric interference filters composed of alternating layers $H$ and $L$. The shorthand notation for this device is $A(H L)^{k} H L L H(L H)^{l} A$, with $k, l=0,1,2, \ldots$. The Fabry-Perot etalon suppresses all radiation within its stopband except for a narrow band centered at $\lambda_{0}$. Its quality is growing with increasing reflector efficiencies, i.e. when the index contrast and/or the number of layers in each reflector increases. This filter is shown in Fig. 2 and its Reset sequence is A HLHLH $2 L$ HLHLH A.

\subsection{Inverted Fabry-Perot etalon}

This is a variation of the Fabry-Perot etalon featuring the dielectric resonator $H H$. It is described as $A(H L)^{k} H H(L H)^{l} A$ and resetted to A HLHLHL $2 H$ LHLHLH A.

\subsection{Weakly coupled Dual cavity filter}

The Dual cavity filter is made of two coupled resonators $\mathrm{HH}$ and $L L$ separated by a reflector and placed between two high quality reflectors. It is described by a formula $A(H L)^{k} H H(L H)^{l}$ $L L(H L)^{m} H A$. Its transmittance spectrum has two narrow peaks shifted relatively to $\lambda_{0}$ inside of the filter stop-band. The splitting amount depends on the strength of resonators coupling. Consequently the splitting decreases with increasing quality of the central reflector. The default configuration is A HLHL $2 \mathrm{H} \mathrm{LHLH} 2 \mathrm{~L}$ HLHL HA. 


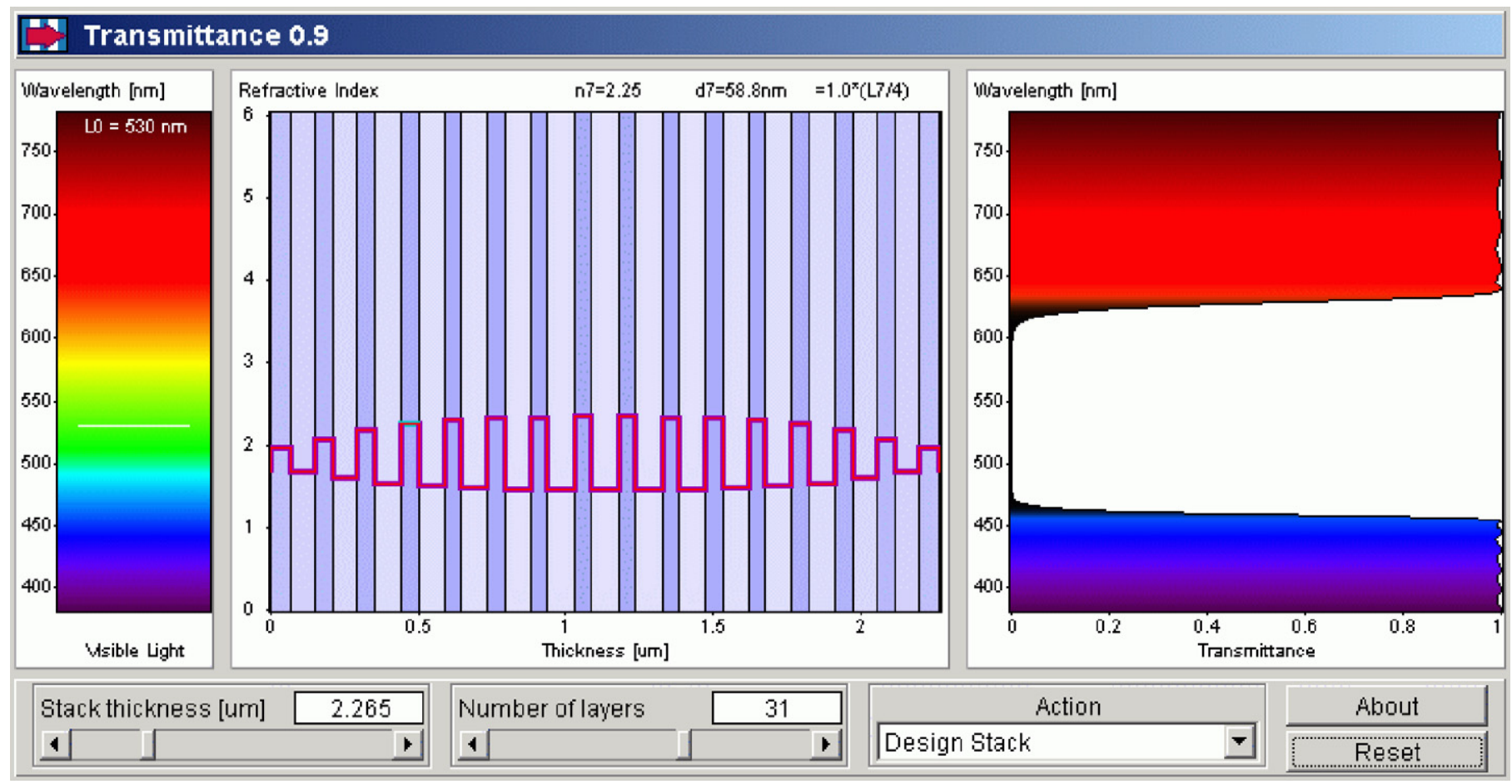

Fig. 3. The 31 layer equal ripple Chebyshev interference filter.

\subsection{Strongly coupled Dual cavity filter}

This filter does not have a central reflector. It is described as A $(H L)^{k} H H L L(H L)^{m} H A$ and has a constant coupling strength between the two resonators. As a result both the splitting and positions of transmission peaks are nearly constant within the stopband. Increasing the efficiency of the left and right reflectors leads to a narrowing and a slight shift of these two peaks. The default configuration is A HLHLHL $2 \mathrm{H} 2 \mathrm{~L} \mathrm{HLHLHL} H$ A.

\subsection{Periodic stack}

This is a simple periodic dielectric structure $(h l)^{k}, k=1,2,3, \ldots$. But now we do not require that layer thicknesses modulo quarterwavelengths are zeros. Notations $h$ and $l$ stand here for dielectric layers of high- and low-index materials with arbitrary optical thicknesses. The central wavelength $\lambda_{0}$ does not have a definite meaning for this type of dielectric stacks. It can however serve as a reference for physical interpretation of the layer thickness in terms of selected quarter-wavelength $\lambda_{0}$. The Periodic stack opens a broad field of experimentation with the interference properties of multilayer dielectric stacks. It is resetted to the short-wave-pass filter $A 0.8 *(2 H L)^{6}$ A.

\subsection{Design stack}

This stack has no restrictions on refractive indices and thicknesses for any dielectric layer. They can be easily modified using the mouse. An irregular stack with many layers can have a complicated spectral response, so the definition of layer thickness in terms of quarter-wavelengths can give useful hints for the stack design and for tracing the origin of resonances on the transmittance curve. Generally it is easier to start from one of the above mentioned filters or etalons and modify their parameters in this design mode. For the design stack, the Reset button cycles the Applet through a set of seven state-of-the-art filters:
- Equal ripple 31 layer Chebyshev sequence $G \prod_{i=1}^{31} M_{i} \quad G$. It is shown in Fig. 3 and has periodically repeated bands of maximum and minimum transmittance subjected to fluctuations of a constant amplitude [2].

- Equal ripple 15 layer Chebyshev sequence $A \prod_{i=1}^{15} M_{i} M$. This filter behaves in a way similar to the previous one but its transmittance achieves only $70 \%$ of the maximal value [2].

- Narrow band-pass filter A HHL $H^{3} L H(H L)^{3} H(H L)^{3} H H L$ $H^{3}$ LHH A. This high quality filter [3] is composed of several coupled cavities separated by reflectors. It provides efficient light suppression outside its pass-band.

- Equal ripple asymmetric narrow band-pass filter $A(L L L M H)^{2}$ $(L H M H L)^{2}(M H H L H)^{2}(L H M H L)^{2}(L L L M H)^{2}$ A. It illustrates that desired filtering properties can be achieved not only with symmetric repetition of $H, L$ layers [2].

- Short-wave-pass edge filter [2] made of slabs with variable thickness, as $A 1.12(L / 2 H L / 2) 1.06(L / 2 H L / 2) \quad 1.03(L / 2 H L / 2)$ $1.015(L / 2 H L / 2)(L / 2 H L / 2)^{3} 1.015(L / 2 H L / 2) 1.03(L / 2 H L / 2)$ $1.06(L / 2 H L / 2) 1.12(L / 2 H L / 2) G$.

- Typical long-wave-pass filter $G(H / 2 L H / 2)^{9}$ A [5].

- Five-cavity wide band-pass filter [11] with unequal spacers, $G$ $H L H 2 L(H L)^{3} H 4 L(H L)^{3} H 6 L(H L)^{3} H 4 L(H L)^{3} H 2 L$ $H L H$ G. It demonstrates the elegant utilization of cavities on unequal optical length in designing high quality optical filters with extended bandwidth.

\section{Conclusion}

We have presented a highly interactive Java applet for real time modeling and visualization of multiple interference transmittance filters. The Applet has been created to supplement the vast literature devoted to this problem which finds extended applications in science and engineering.

The interference filters are realized as multilayer stacks of thin dielectric films with selected thicknesses and refractive indices. The parameters of these stacks can be interactively adjusted by means of the Applet's graphical user interface. The finalized model is processed in real time by the mathematical 
kernel of the Applet which is based on the transfer matrix formalism and implemented in Java. The corresponding transmission spectrum is immediately available for visualization and experimentation. Our program can efficiently demonstrate the most important multiple interference phenomena leading to the formation of the filter's pass- and stop-bands. It is useful to further our understanding of light propagation and scattering in stratified media.

\section{References}

[1] H.A. Macleod, Thin-Film Optical Filters, second ed., Adam Hilger Ltd., 1986.

[2] A. Thelen, Design of Optical Interference Coatings, McGraw-Hill, Inc., 1989.

[3] P. Baumeister, Design of a wavelength-division multiplexing bandpass with quasi-Chebyshev spectral shape, Applied Optics 40 (2001) 1132-1137.
[4] R.K. Sinha, S. Garg, K.L. Deori, Design of a thin-film-based optical filter for broadband multichannel communication systems, Czechoslovak Journal of Physics 53 (2003) 417-424.

[5] W. Chen, P. Gu, Design of non-polarizing color splitting filters used for projection display system, Displays 26 (2005) 65-70.

[6] F. Esquembre, Easy Java Simulations: a software tool to create scientific simulations in Java, Computer Physics Communications 156 (2004) 199-204.

[7] J. Martin, L.N. Long, Using Java for scientific programming and electromagnetics, Computers in Physics 12 (1998) 601-607.

[8] R. Granlund, E. Berglund, H. Eriksson, Designing web-based simulation for learning, Future Generation Computer Systems 17 (2000) 171-185.

[9] J. Hurkała, M. Gall, R. Kutner, M. Maciejczyk, Real-time numerical simulation of the Carnot cycle, Eur. J. Phys. 26 (2005) 673-680.

[10] F.H. Fenton, E.M. Cherry, H.M. Hastings, S.J. Evans, Real-time computer simulations of excitable media: Java as a scientific language and as a wrapper for $\mathrm{C}$ and FORTRAN programs, BioSystems 64 (2002) 73-96.

[11] P. Baumeister, Bandpass filters for wavelength division multiplexingmodification of the spectral bandwidth, Applied Optics 37 (1998) 6609-6614. 